\title{
Detection of Lotus Root Contaminants Using Intelligent Visual Machine Vision Techniques
}

\author{
Yuan Hao \\ School of Mechanical Engineering \\ Jiangsu University \\ Zhenjiang, Jiangsu 212013, China \\ Samuel B. Wilson (Corresponding author) \\ School of Mechanical Engineering \\ Jiangsu University \\ Zhenjiang, Jiangsu 212013, China \\ Emmanuel Asamoah \\ School of Mechanical Engineering \\ Jiangsu University \\ Zhenjiang, Jiangsu 212013, China \\ Cai JianRong \\ School of Mechanical Engineering \\ Jiangsu University \\ Zhenjiang, Jiangsu 212013, China
}

Bao XuKang

School of Mechanical Engineering

Jiangsu University

Zhenjiang, Jiangsu 212013, China 
URL: http://dx.doi.org/10.5296/jfi.v5i1.17813

\begin{abstract}
Lotus root, which is the most important aquatic vegetable in China, is manually inspected for quality by experts to detect impurities in a food production plant in China. There is therefore the need to update this inspection process in order to improve the quality and safety of lotus root. Machine vision systems and techniques are used for consistent, efficient, effective, and reliable inspection of images. This technology has helped several industries in their effort to visually inspect and analyze products. The lotus root inspection system has been proposed to inspect the lotus roots for impurities. The detection algorithms use the size, shape, texture and color of the lotus root and impurities images as parameters to analyze the quality of lotus roots. The lotus root undergoes some cleaning processes before image acquisition and image processing. The camera and illumination used, in collaboration with the edge detection, and image segmentation techniques, efficiently and effectively exposed the impurities in the lotus root at a much faster rate. This proposed inspection technique is less expensive, effective and more efficient compared to the traditional human inspections.
\end{abstract}

Keywords: Machine vision techniques, safety, Image processing, Inspection, Impurities, detection

\title{
1. Introduction
}

Lotus root is one of the most widely consumed water plants in the Asian continent. It is also known as the most important aquatic vegetable in China ( $\mathrm{Lu}$ et al., 2017). They are mainly located in China, India, Korea, and Japan and are greatly cherished by the Chinese and the Japanese (Park \& Lee, 2020). Due to the massive consumption and the economic benefits of lotus root, it is very important to ensure the quality standards of its production (Min et al., 2021). In the past decades, manual inspections were used by agricultural quality experts to check the quality of produce (Alçiçek \& Balaban, 2012). A food production company located in China, which produces steamed lotus root stuffed with sweet sticky rice, uses lotus root in their food processing production line. The inspection process of this company is done by inspection experts. This process is highly inefficient because it takes about 30 seconds to a minute for the inspector to fully assess and inspect each lotus root and there is the possibility of human error and fatigue. The recent use of machine vision techniques in various food and agricultural sectors has proven to be more effective, time saving and less costly. Therefore, there is a need to apply this technology and these techniques in food production in order to improve productivity and save time and money. Recently, the machine vision technology has been widely adapted into the agricultural sector to inspect the quality of food produced by farmers before it was sent to the market for consumption (Vision, 2019). Machine vision systems have been proven to be the best technology to inspect the quality of food (Iraji, 2019), because of their accurate and efficient results compared to the traditional method, where the inspections are done manually by experts ( $\mathrm{R}$. Gao \& Wu, 2015). The main focus of this food 
production line and many other food processing industries are food safety and hygiene (Garcia, Osburn, \& Jay-Russell, 2020; Sikora \& Strada, 2005).

Roy et al., (Roy, Chaudhuri, Bhattacharjee, Manna, \& Chakraborty, 2019) in their research to sort out fresh vegetables from the rotten ones developed image segmentation techniques to enable them achieve the desired results. In their study, Marker Based Segmentation, Color Based Segmentation, and Edge Detection were the techniques used to detect the rotten vegetables. The results of the used techniques showed much better efficiency. The Marker Based Segmentation basically use the pixels of the captured image and analyzes them. Conversely Color Based Segmentation is mainly based on the differential color and the position of the camera since change in position of the camera will have the results affected due to light reflections. The used segmentations algorithms and edge detection technique was able to effectively identify and detect the rotten parts of the vegetables. The results aided in classifying the rotten vegetables from the healthy ones. Of all the techniques, the edge detection was the most effective and efficient. Momin and colleagues (Momin et al., 2017) in their studies they used global thresholding color binarization, combined with median filter and morphological analysis to automatically grade the mangos for the post-harvest processes. This was a huge relief for most mango farmers. This post-harvest process was usually done manually by experts so the proposed research was welcomed by most of the farmers in Bangladesh. The grading system which was developed graded the mangos into sizes i.e., small (below 150g), medium (150-300g) and large (over 300g). The developed algorithm used area, perimeter and roundness as parameters for the grading of the mangos. At the end of their studies, the results of the analysis on the mass of mangos, feret diameter and perimeter proved more efficient than the results of the analysis based on the perimeter and roundness. Ramirez-Paredes and his partner (Ramirez-Paredes \& Hernandez-Belmonte, 2019) investigated the quality assessment of malting barley using image processing technology. They employed color, shape and texture as their parameters for the detection algorithm to check the quality of the malting barley grains.

In this study, we shall report on the investigation of detecting impurities in lotus root. The purpose of this study was to use machine vision technology and techniques to ensure food hygiene and safety in order to aid a food production line in China that uses lotus root in its production. This is to improve food safety and productivity, and also save money and time. Figure 3 shows the $3 \mathrm{D}$ of the production line where the machine vision technology and technique will be incorporated. We proposed four image processing algorithms to detect impurities based on differential pixel and gradient intensities of lotus root and impurities. The parameters such as; color, shape, texture and size were taken into consideration to develop machine vision techniques to perform the detection and inspection process.

\section{Materials and Method}

\subsection{Lotus Root}

Lotus roots is a water plant cherished by people in the Asian continent and some other parts of the world (H. Gao, Chai, Cheng, \& Cao, 2017). The objective of this research is to detect the impurities which could be present in the lotus root. One possible variable that may affect 


\section{Macrothink

the results of this experiment is the polyphenol compound in lotus root which makes it turn brown easily when oxidized (J. Li et al., 2020). To avert this from affecting the results of the experiment, different approaches are taken to prevent the oxidation of the lotus root slice. Ali and colleagues (S. Ali et al., 2020) in the research treated the lotus root slice with oxalic acid and kept at $20 \pm 1{ }^{\circ} \mathrm{C}$.

The lotus root undergoes some preservation processes before the inspection of the lotus root by the machine vision techniques. The lotus root is initially cleaned and washed with cool fresh water. The outer skin is peeled and the lotus root is sliced. The lotus root is cut into the dimensions of 30-35 mm slices. The slices are soaked in rice vinegar diluted with water to prevent the browning of the lotus root. After cleaning and soaking in the rice vinegar, the sliced lotus root is frozen at a temperature of minus one to three degrees Celsius $\left(-1\right.$ to $\left.3^{\circ} \mathrm{C}\right)$ before undergoing the developed machine vision techniques and algorithms. Lotus root can be stored for about four days until it is requiring food processing. We analyzed the lotus root images in this particular research to detect for the possible presence of impurities.

\subsection{Vision System}

An allied vision camera, Mako G-507C was used to capture images of the lotus root sections. It has maximum quality Type 2/3 (11.1 mm diagonal) Sony IMX264 CMOS sensor incorporated in it. This apparatus was very affordable and easy to acquire. The camera runs 23.7 frames per second when the resolution is at its maximum. Figurel shows the experimental setup for this study. The camera was connected to the computer to take images of the sample lotus root, and the images were analyzed with the computer using the developed algorithms.

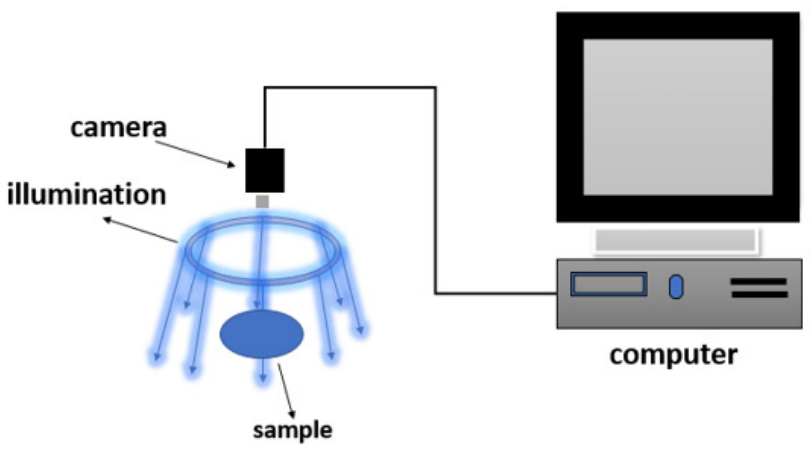

Figure 1. The machine vision experimental setup for lotus root image acquisition and analyzation 


\section{MInstitute ${ }^{\text {Mink }}$}

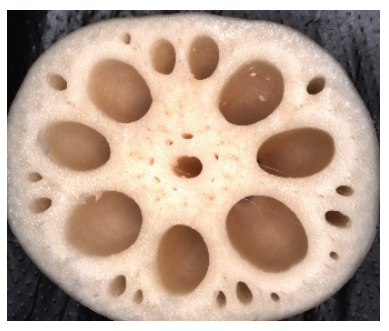

(A)

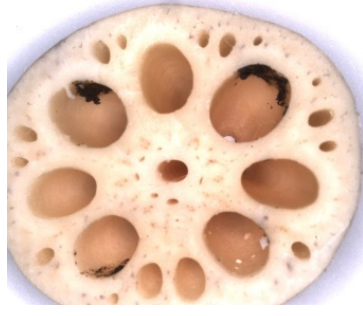

(B)

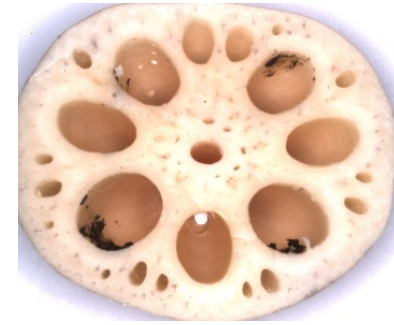

(C)

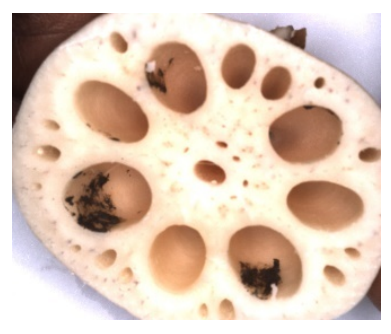

(D)

Figure 2. Some lotus root images taken by experimental setup in Figure 1. (A) has a black background which eliminates the shadows in the image and (B), (C), and (D) are with white backgrounds and the shadows can be seen

The inner part of the illumination box in our setup was covered with a black linen to reduce the shadows and lights that might reflect in the background. A white circular coaxial LED light source was used and fitted at the top of the containment to illuminate the lotus root. This lighting evenly exposed the lotus root for segmentation (J. M. Ali, Jailani, \& Murugan, 2019).

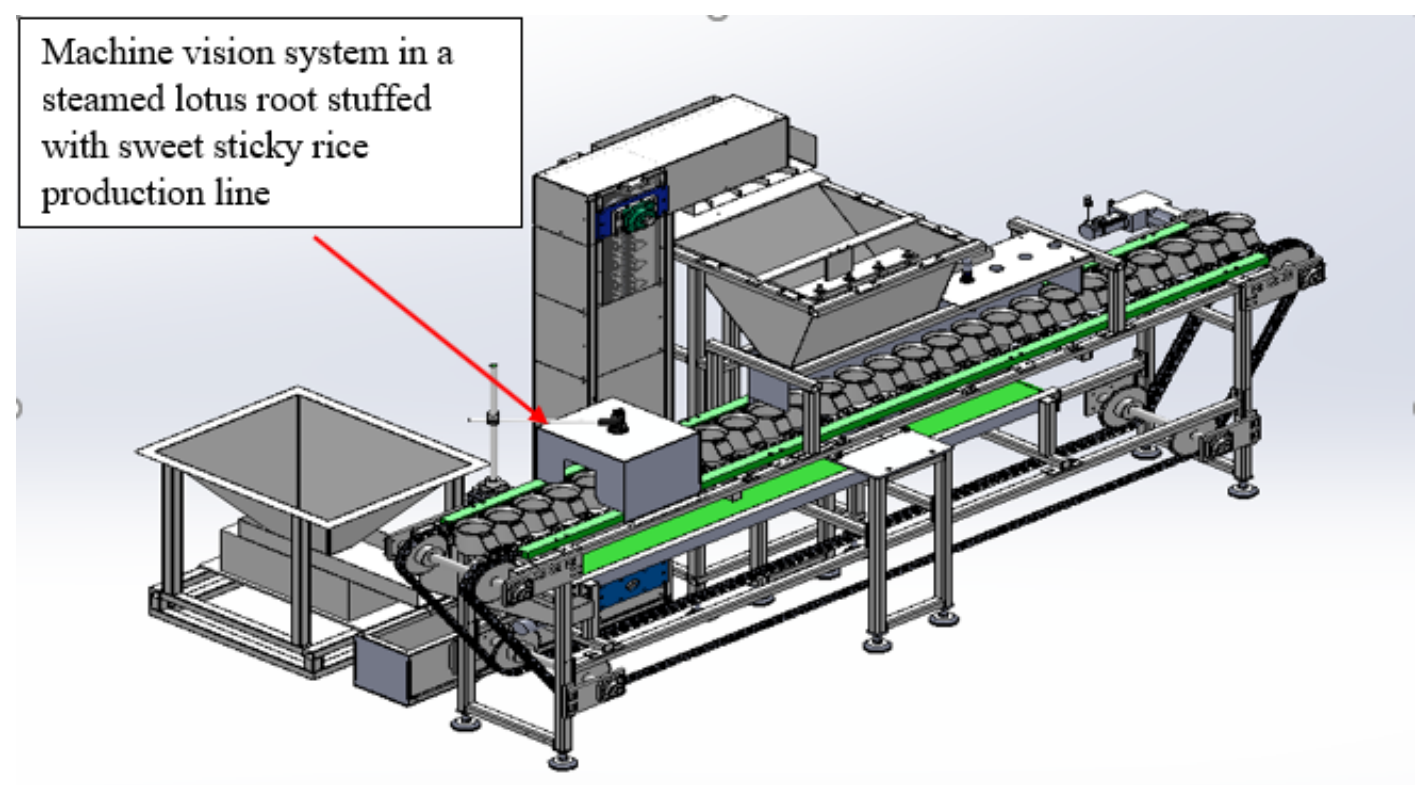

Figure 3. Production Line of steamed lotus root stuffed with sweet sticky rice where the Machine Vision Technology will be employed to inspect the lotus root for impurities

\subsection{Image Analysis}

The developed algorithms are used to detect for the presence of impurities in the lotus root, and the parameters taken into consideration to analyze the images are; size, shape, texture and color. This program can be manipulated to alter the value of the threshold used to binarize the image. The thresholding and region segmentation machine vision technique uses the color and texture of the impurities and lotus root to segment and expose the impurities in the lotus 


\section{Ml Macrothink}

root due to the differential pixel intensities. The images are segmented into background and foreground regions due to the differential pixel intensities. The shape, color, texture and size mostly determine the intensity gradient of the pixels. Due to the differential gradient intensities of the lotus root and the impurities, the edge detection algorithm is able to cause formation of the edges of the impurities in the lotus root by hysteresis.

Using OpenCV $\mathrm{C}++$, the proposed algorithms load the lotus root image captured by the camera and then analyze the images to detect the presence of impurities. The algorithm resizes the image to a desired size and then converts it to grayscale image. Figure 4(a) shows the image of the lotus root before undergoing grayscale analysis and Figure 4(b) represents the grayscale image obtained after running the algorithm.

(a)

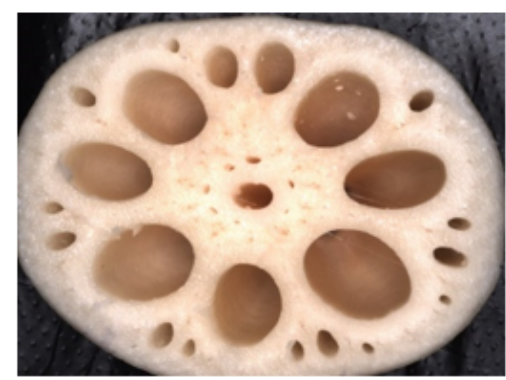

(b)

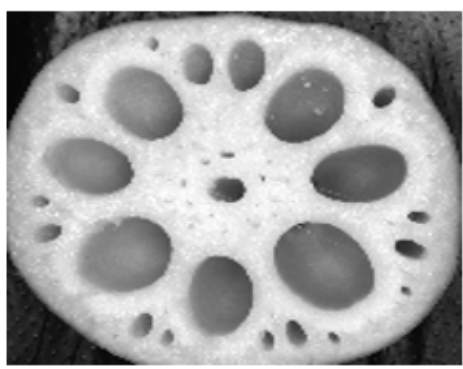

Figure 4. (a) The original image of the lotus root before the gray-scaling and (b) the image obtained after the grayscale analysis

After changing the lotus root image to grayscale, the denoising algorithm is used to remove noise and blurs from the image and the necessary machine vision techniques and algorithms are applied.

\subsection{Impurities Detection Analysis}

According to the research by Shrivakshan and Chandrasekar (Shrivakshan \& Chandrasekar, 2012), there are two main approaches in determining the edges of an image, that is the gradient and the Laplacian method. In this stage, an edge detection algorithm using Canny filters was develop to detect the boundaries of impurities present in the lotus root. The algorithm is able to detect the edges of the impurities in the holes of the lotus root due to the differential pixel intensity gradients of the impurities and the lotus root. The algorithm first resizes the image to $500 \times 500$, and then converts it into a grayscale image. The grayscale image is denoised to remove blurs and increase sharpness and the gradient intensity of the image is determined. The next step is application of non-maximum suppression to eliminate sections that cannot form edges, followed by hysteresis thresholding. The minimum threshold was set at 0 and the maximum 100. To achieve the best edge detection, the Canny Threshold value can be set between 80 to 95 . In this study, we used the threshold value of 85 because it effectively eliminated weak edges and exposed the edges of the impurities in the lotus root. Figure 6 shows the results of the captured lotus root images after it has undergone the edge detection analysis. 


\section{Macrothink}

The developed adaptive threshold algorithm is determined by a small region around the target image Local adaptive thresholding is employed to determine the threshold value since it proved to be efficient more than the Chow and Kaneko approach (Jyothi \& K.Bhargavi, 2014). The program loops through each pixel, finds the mean of its immediate $5 \times 5$ neighbors (i. e. the neighboring pixels with the main one in the middle that forms a $5 \times 5$ matrix). Then the program compares each pixel within the $5 \times 5$ matrix with the calculated mean. The algorithm scrutinized other neighboring pixels' image intensity to segment the image into two regions. The threshold value, which was used to develop the adaptive threshold algorithm to be able to segment the impurities from the lotus root, considered the differential pixel values to be either 0 or 255 (Shafait, Keysers, \& Breuel, 2008). Therefore, when the threshold is greater than the pixel value it forms the background, in turn when the threshold is lesser, it forms the foreground. The algorithm took less than 2 seconds to analyze an image. Figure 8 is the outcome of the lotus root images that went through adaptive threshold analysis.

The next inspection algorithms developed to inspect the lotus roots were the image segmentation algorithms. This research used adaptive and global threshold segmentation, and region growing segmentation to differentiate the impurities from the lotus root. With the image segmentation, the image is initially resized to 500x500 pixels. After denoising the image, the developed adaptive threshold analyzes the lotus root image by dividing it into sub-images and the threshold for each sub-image is calculated. It loops through all the image pixels and separates them into Red, Green and Blue channels. The algorithm then calculates the gray weighted value using the formula $0.299 *$ Red channel $+0.587 *$ Green channel + $0.114 *$ Blue channel. Then the gray weighted value calculated for each pixel is assigned at the same coordinate position as the source image to an image variable. The resulting image is then binarized.

The developed Global threshold algorithm divides the image into two regions that is background and foreground, lotus root and impurities respectively by a single threshold. The formula used for the global thresholding is;

$$
g(x, y)=\left\{\begin{array}{l}
1, \text { for } f(x, y)>T \\
0, \text { for } f(x, y) \leq T
\end{array}\right.
$$

With $g(x, y)$ being the thresholded image and $T$ the threshold value, which is constant for the whole image. The algorithm assumes the image to have bimodal histogram. The developed algorithm use Hue, Saturation and Intensity (HSI) and Red, Green and Blue (RGB) in its evaluation for thresholding because of the color difference of the impurities and lotus root (Gonzalez, Woods, \& Eddins, 2004). The acquired images are transformed to HSI from RGB and then to grayscale. The pixels of the impurities and lotus root have their gray levels grouped into two dominant modes, the white and dark modes respectively. The differential pixel intensities of the lotus root image enable the algorithm to analyze and successfully differentiate the impurities and the lotus root. To extract the impurities from the lotus root, threshold $T$ is selected to separate these modes. The threshold value is set as 127 (Rogowska, 2000). If the pixel intensity value between the two modes is lower than the threshold, it forms 
the foreground, i.e., the impurities, also known as the white pixel. However, if the pixel intensity is higher than the threshold, it forms the background, that is the lotus root, with the dark pixel (Shao et al., 2019). Figure 10 show the lotus root after global thresholding analysis.

The final algorithm developed for this work was the region growing segmentation algorithm. The foundation for region growing segmentation is seed growing. The developed segmentation algorithm employs color, texture, shape, size, motion, average intensity and variance as characteristics in its region determination. In this segmentation technique, the pixels of neighboring initial seed points are examined and checked to determine whether it qualifies to be part of a particular region. To achieve the best outcome as seen in figure 12, good parameters should be chosen to determine the blob, that is the impurities in the segmentation analysis. This algorithm iteratively blends the pixels or image patch areas by conserving a minimal cost of the overall search, the process can be summarized as:

1. $\mathrm{P}_{\text {seed }}$, is considered as seed pixel, taking into consideration the neighboring pixels, and the pixel(s) with the least intensity difference from $\mathrm{P}_{\text {seed }}$ will be included in the initial pixel pool, the blob.

2. Calculations of the mean $\mu$ and standard deviation $\sigma$ of the pixels are carried out.

3. As shown in figure 11 and 12 , assume that there are a set of $\mathrm{N}$ pixels, $\mathrm{P}=\left\{\mathrm{P}_{1}, \ldots, \mathrm{P}_{\mathrm{N}}\right\}$, on the outside boarder of the current blob, then each pixel in the set $\mathrm{P}$ are subjected to a similarity test, which determines whether the pixel should be included in the blob. The similarity measure for pixel $\mathrm{P}_{\mathrm{i}} \in \mathrm{P}$, is defined as statistical distance $\mathrm{D}_{\text {stat }}=\left(\mathrm{P}_{\mathrm{i}}-\mu\right) / \sigma$

Where $\mathrm{P}_{\mathrm{i}} \in \mathrm{P}$ has the pixel intensity $\mathrm{p}_{\mathrm{i}}, \mu$ and $\sigma$ are the mean and standard deviation of the blob. Only the pixel(s) with minimum $\mathrm{D}_{\text {stat }}$ will be added to the blob. A predefined threshold $D_{\max }$ starts here, where pixels with $\mathrm{D}_{\text {stat }}$ greater than $\mathrm{D}_{\max }$ will make it unsuitable for blob membership. If there is no pixel's inclusion of $\mathrm{P}$ in the blob, the region growing algorithm stops, otherwise steps 2 and 3 will repeat.

When region growing stops for every seed pixel in the image, blob features including mean and standard deviation are extracted and will be used to identify impurities in the lotus root. It takes less than 3 second for the algorithm to segment the loaded image.

\section{Results and Discussion}

The sliced lotus root was placed in an illumination box with the inner walls blackened. The white coaxial light used gave the right illumination for the camera to be able capture good images of the lotus root. Mirante and his team (Mirante, Georgiev, \& Gotchev, 2011) used the same device to develop their vision system for fast image segmentation algorithm for a color and depth map. The study of González-Rivas and his colleagues, (González-Rivas, Ripolles-Avila, Fontecha-Umaña, Ríos-Castillo, \& Rodríguez-Jerez, 2018) revealed that a good illumination setup advances the analysis of the captured image. The lighting used had a major impact on the ability of the algorithms to detect the impurities (Vafeiadis et al., 2018). 


\section{Mll Macrothink}

Visual studio 2017 was used alongside with OpenCV C++ to write the algorithms for the image processing. All of the developed algorithms took between 2 to 3 seconds to successfully analyze an image. This gave a satisfactory result of the machine vision technique used being more effective and efficient than the manual inspection method. The developed algorithm was used to analyze about 200 lotus root images. The images below show few outcomes of the undertaken experiment. Figure 4 is the initial stage of the image processing where the image is changed to grayscale. Before an image can undergo canny edge detection and image segmentation, it has to be changed to black and white to represent the amount of illumination. Grayscale conveys only the intensity information of the image. A study from the University of Auckland, New Zealand, showed Gaussian blur algorithm performed on the grayscale images reduces any noise the image may have.

We developed the edge detection algorithm to analyze the captured lotus root images. The edge detection algorithm used color, shape, and size as parameters to analyze the captured lotus root images. The shape, color, texture and size are mostly determined by the intensity gradient of the pixels using Canny edge detection (Meng, Zhang, Yin, \& Ma, 2018). The algorithm first changed the lotus root image to grayscale, denoised the image by suppressing the noise without causing damage to the edges and then enhanced the quality of the edges. The gradient intensity of the lotus root image was calculated and non-maximum suppression was applied. The final process was hysteresis. The developed algorithm was able to achieve the desired results due to the differential gradient intensity of the impurities and the lotus root and also the threshold value selected. The edges of impurities were outlined by lower and upper thresholds. If the pixel gradient intensity is higher than the upper threshold, it is considered as an edge but if less than the lower threshold, it is rejected. If pixel gradient is found in between the two thresholds, it is only accepted when it is connected to a pixel that is above the upper threshold (Kneip, Fleischmann, \& Berns, 2020). It took the algorithm less than 3 seconds to effectively analyzed an image. The results are shown in Figure 5 and Figure 6.

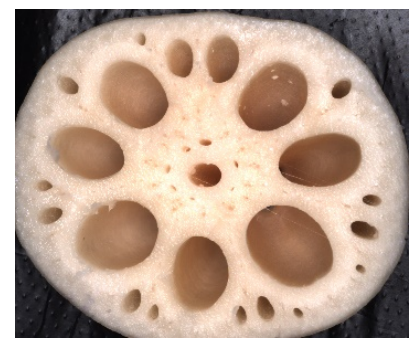

(A)

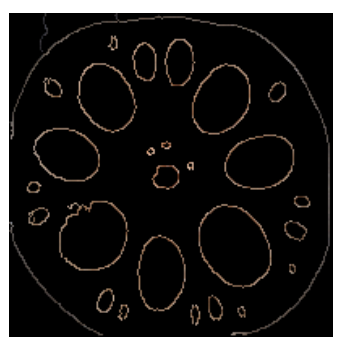

(B)

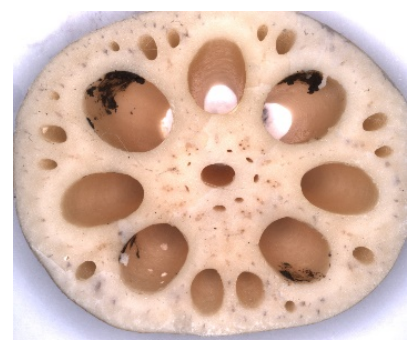

(C)

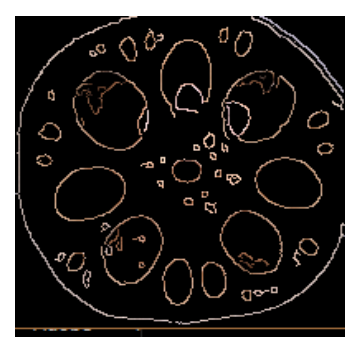

(D)

Figure 5. (a) shows the captured lotus root image without impurities with a black background and the result after undergoing edge detection is shown in (b), (c) is the lotus root image with impurities having a white background, and the results after edge detection analysis is shown in $(d)$ 


\section{Macrothink}

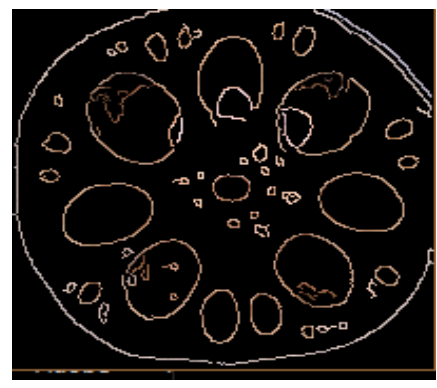

(A)

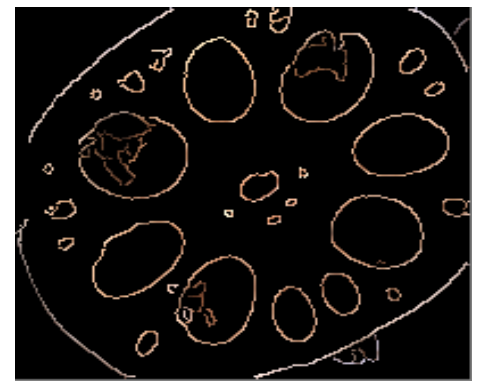

(B)

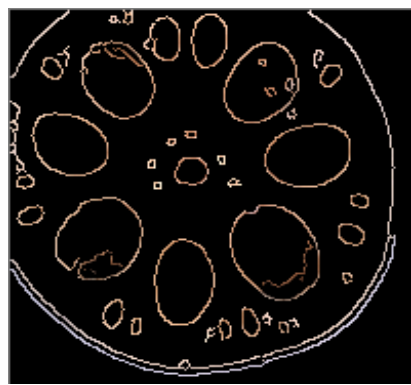

(C)

Figure 6. (a), (b), and (c) are images of the lotus root that have undergone the edge detection technique. The outlines of the impurities in the lotus root are detected as edges

In other to achieve best detection outcome, image segmentation algorithms, that is global and adaptive thresholding, and region growth segmentation were also developed to analyze the image. The images are segmented into background and foreground regions (Y. Li et al., 2019). The image segmentation algorithm was used to group pixels into salient image sections, that is regions corresponding to individual surfaces, objects, or natural parts of objects. Image Segmentation uses the differential pixel intensities of the impurities and the lotus root to analyze the image.

The adaptive threshold algorithm developed solved the greyscale image and divided the image into sub-images (Vitzrabin \& Edan, 2016), then the threshold for each sub-image was calculated (Zemmour, Kurtser, \& Edan, 2019). A small region around the target image is used by the developed technique to analyze and differentiate the foreground and background (Kumar, Dubey, \& Jothi, 2017). The pixel intensities are calculated and if the value is below the threshold, it forms the background i.e., the lotus root and if the intensity value is above the threshold, it forms the foreground, i.e., the impurities as seen in figure 8 and $7(\mathrm{~d})$. According to Wang and his team, (Wang, He, Han, Ouyang, \& Li, 2013), the results of adaptive threshold are sometimes better compared to that of global thresholding. The algorithm was able to detect the impurities in the lotus root due to the differential pixel intensities of both the impurities and the lotus root. Also Li et al., (D. Li, Xu, \& Liu, 2017) research examined image intensity of neighboring pixels to determine adaptive threshold. 


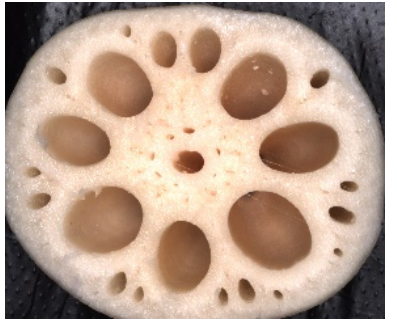

(A)

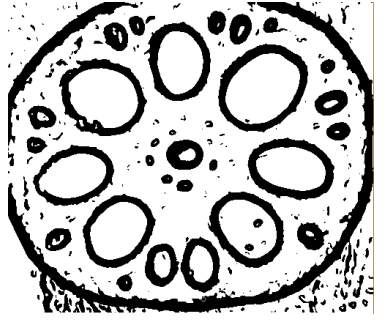

(B)

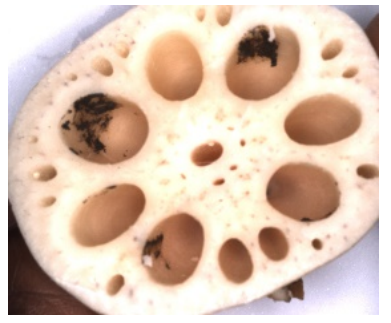

(C)

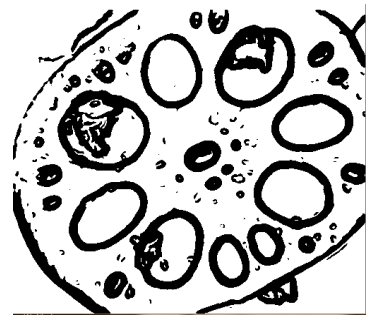

(D)

Figure 7. (a) The image of lotus root without impurities with black background and the result is (b) after (a) undergoing adaptive thresholding. (c) The lotus root image with white background with impurities and the results after it had undergone adaptive threshold is shown in (d).

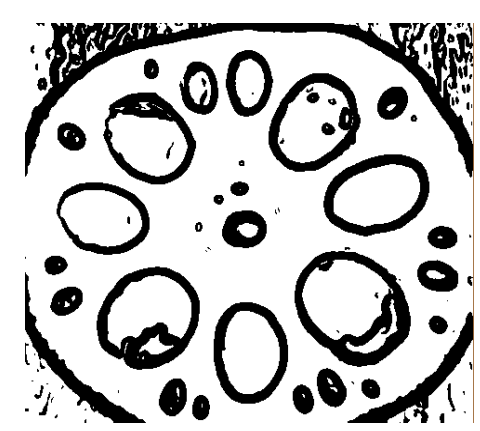

(A)

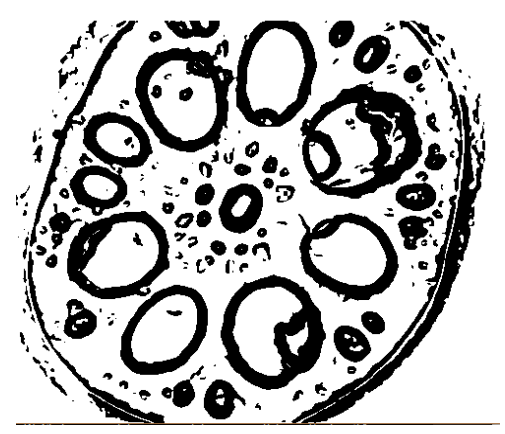

(B)

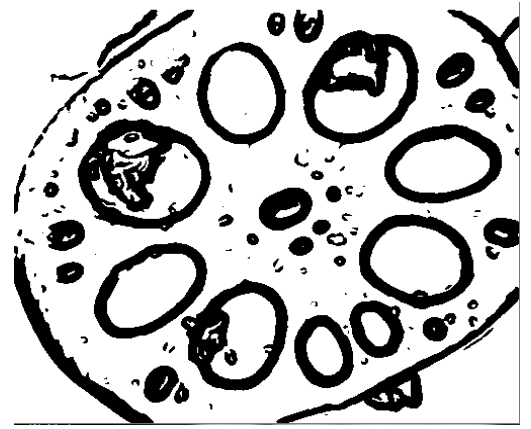

(C)

Figure 8. (a), (b), and (c) are results of lotus root images that have undergone adaptive thresholding

The global threshold algorithm used a single threshold to group the image into background and foreground regions. Where the lotus root is the black background and the impurities is the white foreground. This machine vision technique assumes the image to have bimodal histogram (Mavridou, Vrochidou, Papakostas, Pachidis, \& Kaburlasos, 2019). The intensity of a pixel varies between 0 to 255 . If the minimum threshold value between the two modes is lower than the pixel intensity, it forms the foreground but if it is higher than the pixel intensity, it forms the background as shown in figure 10 and 9(d). Ruedt et al., (Ruedt, Gibis, \& Weiss, 2020) used global threshold to successfully segment iridescent sections of meat. Not only was their system less expensive and easier to operate but also more effective and efficient in its analysis. Momin et al., (Momin et al., 2017) in their studies also used global thresholding color binarization, combined with median filter and morphological analysis to automatically grade the mangos for the post-harvest processes. They studied various image segmentation algorithms and settled on region-based global thresholding color segmentation. Evaluation of Hue, Saturation, and Intensity (HSI) and RGB was done for thresholding. Since 
HSI is superior than RGB, it was ideal for developing the image processing algorithms. At the end of their studies, the results of the analysis on the mass of mangos' feret diameter and perimeter proved more efficient than the results of the analysis based on the perimeter and roundness. The mass of mangos' feret diameter and perimeter gave a success accuracy rate of $97 \%$ whilst that of the perimeter and roundness gave a limited success accuracy rate of (36-79\%). The algorithm used the differential pixel intensities of both the impurities and the lotus root to determine the two regions. It assumed the image to be bimodal and therefore extracted the impurities from the lotus root.

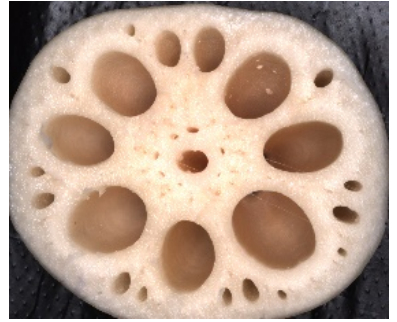

(A)

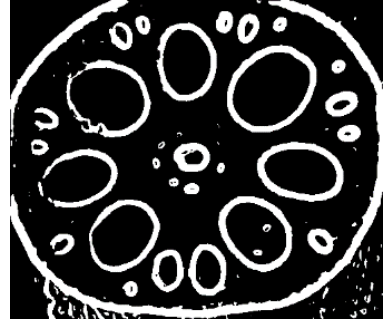

(B)

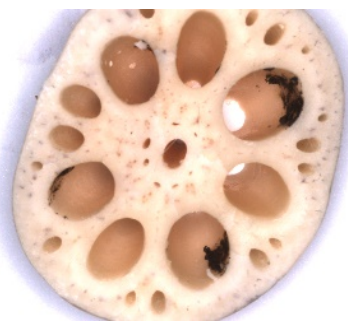

(C)

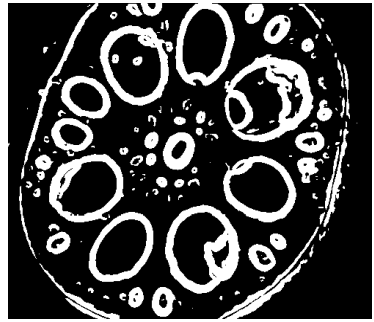

(D)

Figure 9. (a) The image of lotus root without impurities, (b) the results of image (a) after global thresholding, (c) the image of lotus root with impurities and (d) the results of image (c) after global thresholding

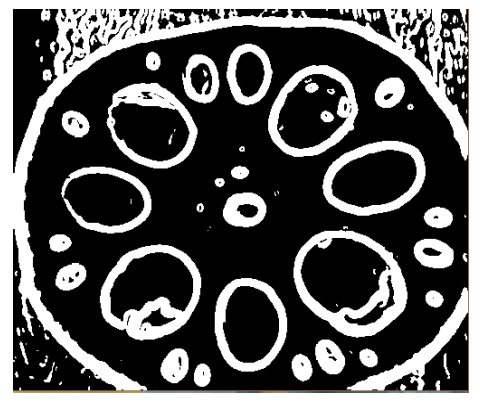

(A)

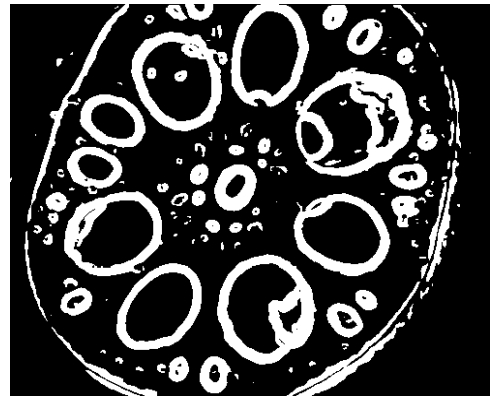

(B)

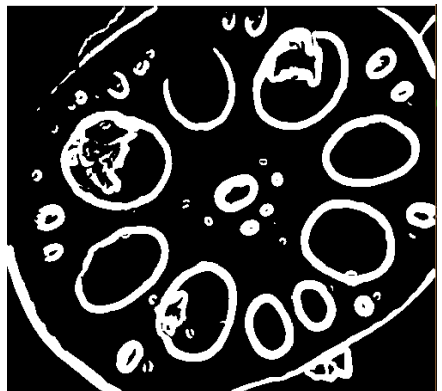

(C)

Figure 10. Figure (a), (b), and (c) are the lotus root images that have undergone the global thresholding algorithm

Region growing segmentation algorithm groups pixels of the same or similar intensities as one region or blob. From Dilpreet and Kaur (Dilpreet \& Kaur, 2014) studies, the segmentation of the image was based on the growing of seeds. Merzougui and Allaoui (Merzougui \& Allaoui, 2019) in determining the region in their research used color, texture, shape, etc. as their parameters to establish the region. It is very simple and performs well with respect to noise. The most important parameter for the algorithm to analyze the image is the predefined threshold, $\mathrm{D}_{\max }$. It ensures the desired object region detection outcome and effectively suppresses false alarms. Figure 11 depicts the outcome of lotus root images taken and analyzed using region growing segmentation algorithm. Jothiaruna et al., (Jothiaruna, 


\section{Mll Macrothink}

Joseph Abraham Sundar, \& Karthikeyan, 2019) established that it is necessary to select good parameters for the blob formation. Their selected parameters were what made the segmentation process accurate and successful. The algorithm first changed the image into grayscale and denoised the image. It then grouped the image into two regions or blobs that is white and black based on the pixel intensities.

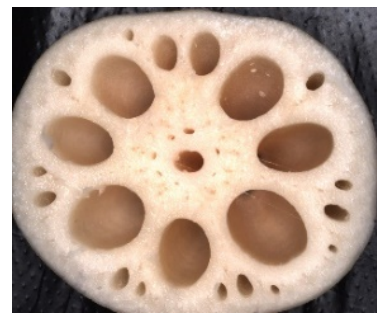

(A)

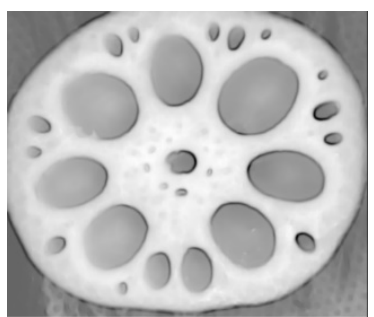

(B)

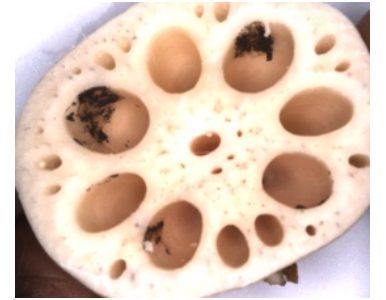

(C)

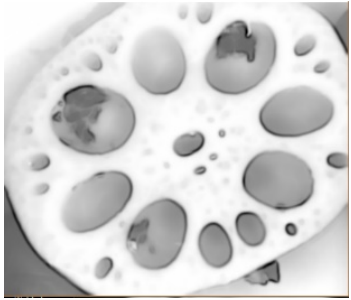

(D)

Figure 11. The image of lotus root without impurities with black background (a), undergoes region growing segmentation and the result, (b). (c) The image of lotus root with impurities with white background and (d) the results after (c) have undergone region growth segmentation

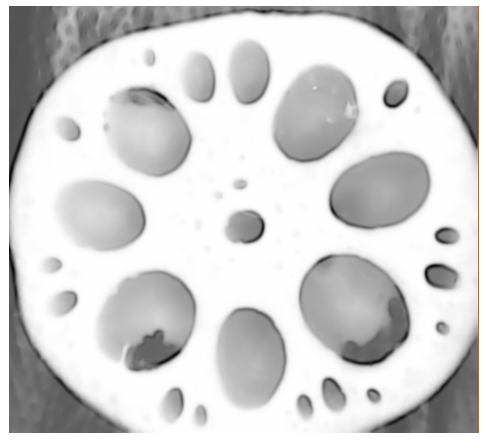

(A)

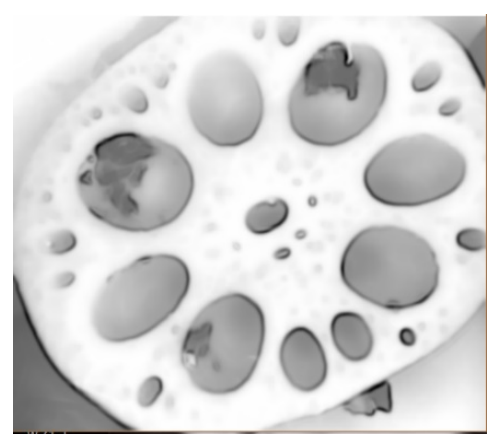

(B)

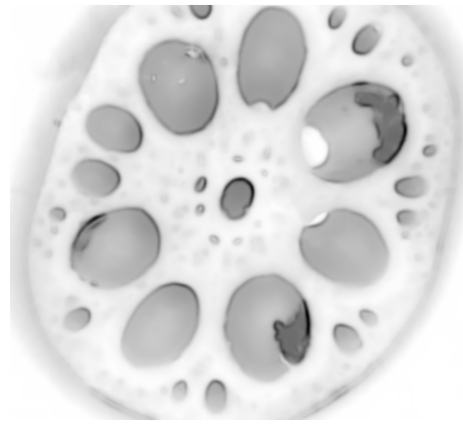

(C)

Figure 12. Different lotus root images with impurities that have gone through region growing segmentation analysis and the e results are shown in (a), (b), and (c)

\section{Conclusion}

This paper aimed to develop a machine vision algorithm which is able to inspect and detect the presence of impurities in lotus root. This method is designed to replace the manual inspection methods which are currently done by experts in food production. The main aim is to improve the inspection process with a faster and less expensive method of inspection for lotus root. It is necessary to put measures in place that ensure food safety and quality before human consumption. The developed inspection algorithms were based on edge detection and image segmentation to differentiate between the lotus root with and without impurities.

The proposed algorithms differentiated between the lotus with and without impurities. The initial machine vision techniques used to analyze the image was the edge detection algorithm, 
after which the global and adaptive thresholding was also used to analyze the image and then finally region growing segmentation algorithm. It took less than 3 seconds when either of the algorithms were used to analyze an image. Comparing it to the traditional method done by inspection experts, all the developed algorithms were faster, more effective, and more efficient. Also, there was less room for error since machines do not succumb to fatigue nor are they susceptible to human errors. The white coaxial LED light aided the developed algorithms to effectively segment the images. After the algorithms had successfully analyzed the lotus root images, we realized the edge detection and region segmentation algorithms performed very well in detecting the impurities, giving a success rate of about 0.99 and both adaptive and global threshold a success rate of about 0.94 . This machine vision technology saves production cost and time compared to the manual inspection done by experts.

\section{Acknowledgments}

This work was supported by the National Natural Science Foundation of China: General Research on Institutional Hypothesis and Function Realization Method Based on the Principle of Rice Suspension Machining, (No. 51975259).

\section{References}

Alçiçek, Z., \& Balaban, M. Ö. (2012). Development and application of "The Two Image" method for accurate object recognition and color analysis. Journal of Food Engineering, 111(1), 46-51. https://doi.org/10.1016/j.jfoodeng.2012.01.031

Ali, J. M., Jailani, H. S., \& Murugan, M. (2019). Surface roughness evaluation of milled surfaces by image processing of speckle and white-light images Advances in manufacturing processes (pp. 141-151). Springer. https://doi.org/10.1007/978-981-13-1724-8_14

Ali, S., Khan, A. S., Anjum, M. A., Nawaz, A., Naz, S., Ejaz, S., \& Hussain, S. (2020). Effect of postharvest oxalic acid application on enzymatic browning and quality of lotus (Nelumbo nuciferaGaertn.) root slices. Food Chemistry, 312, 126051. https://doi.org/10.1016/j.foodchem.2019.126051

Dilpreet, K., \& Kaur, Y. (2014). Various image segmentation techniques: a review. International Journal of Computer Science and Mobile Computing, 3(5), 809-814.

Gao, H., Chai, H., Cheng, N., \& Cao, W. (2017). Effects of 24-epibrassinolide on enzymatic browning and antioxidant activity of fresh-cut lotus root slices. Food Chemistry, 217, 45-51. https://doi.org/10.1016/j.foodchem.2016.08.063

Gao, R., \& Wu, H. (2015). Agricultural image target segmentation based on fuzzy set. Optik, 126(24), 5320-5324. https://doi.org/10.1016/j.ijleo.2015.09.006

Garcia, S. N., Osburn, B. I., \& Jay-Russell, M. T. (2020). One Health for Food Safety, Food Security, and Sustainable Food Production. Frontiers in Sustainable Food Systems, 4(1). https://doi.org/10.3389/fsufs.2020.00001

González-Rivas, F., Ripolles-Avila, C., Fontecha-Umaña, F., Ríos-Castillo, A. G., \& Rodríguez-Jerez, J. J. (2018). Biofilms in the Spotlight: Detection, Quantification, and 


\section{Ml Macrothink}

Removal Methods. Comprehensive Reviews in Food Science and Food Safety, 17(5), 1261-1276. https://doi.org/10.1111/1541-4337.12378

Gonzalez, R. C., Woods, R. E., \& Eddins, S. L. (2004). Digital image processing using MATLAB: Pearson Education India.

Iraji, M. S. (2019). Comparison between soft computing methods for tomato quality grading using machine vision. Journal of Food Measurement and Characterization, 13(1), 1-15. https://doi.org/10.1007/s11694-018-9913-2

Jothiaruna, N., Joseph Abraham Sundar, K., \& Karthikeyan, B. (2019). A segmentation method for disease spot images incorporating chrominance in Comprehensive Color Feature and Region Growing. Computers and Electronics in Agriculture, 165, 104934. https://doi.org/10.1016/j.compag.2019.104934

Jyothi, S., \& Bhargavi, K. (2014). A Survey on Threshold Based Segmentation Technique in Image Processing. 26. K. Bhargavi, S. Jyothi, 3.

Kneip, J., Fleischmann, P., \& Berns, K. (2020). Crop edge detection based on stereo vision. Robotics and Autonomous Systems, 123, 103323. https://doi.org/10.1016/j.robot.2019.103323

Kumar, Y., Dubey, A. K., \& Jothi, A. (2017, 5-6 May 2017). Pest detection using adaptive thresholding. Paper presented at the 2017 International Conference on Computing, Communication and Automation (ICCCA). https://doi.org/10.1109/CCAA.2017.8229828

Li, D., Xu, L., \& Liu, H. (2017). Detection of uneaten fish food pellets in underwater images $\begin{array}{llll}\text { for } & \text { aquaculture. } & \text { Aquacultural } & \text { Engineering, }\end{array}$ https://doi.org/10.1016/j.aquaeng.2017.05.001

Li, J., Bai, J., Li, S., Zhu, Z., Yi, Y., Wang, H., \& Lamikanra, O. (2020). Effect of lactic acid bacteria on the postharvest properties of fresh lotus root. Postharvest Biology and Technology, 160, 110983. https://doi.org/10.1016/j.postharvbio.2019.110983

Li, Y., Sun, R., Liu, Y., Yang, Y., Ma, S., \& Liu, Y. (2019). Interactive foreground segmentation and shape reconstruction from RGBD images. Computers \& Electrical Engineering, 79, 106463. https://doi.org/10.1016/j.compeleceng.2019.106463

Lu, H.-F., Tan, Y.-W., Zhang, W.-S., Qiao, Y.-C., Campbell, D. E., Zhou, L., \& Ren, H. (2017). Integrated emergy and economic evaluation of lotus-root production systems on reclaimed wetlands surrounding the Pearl River Estuary, China. Journal of Cleaner Production, 158, 367-379. https://doi.org/10.1016/j.jclepro.2017.05.016

Mavridou, E., Vrochidou, E., Papakostas, G. A., Pachidis, T., \& Kaburlasos, V. G. (2019). Machine Vision Systems in Precision Agriculture for Crop Farming. Journal of Imaging, 5(12), 89. https://doi.org/10.3390/jimaging5120089

Meng, Y., Zhang, Z., Yin, H., \& Ma, T. (2018). Automatic detection of particle size distribution by image analysis based on local adaptive canny edge detection and modified circular Hough transform. Micron, 106, 34-41. https://doi.org/10.1016/j.micron.2017.12.002 


\section{Ml Macrothink}

Merzougui, M., \& Allaoui, A. E. (2019). Region growing segmentation optimized by evolutionary approach and Maximum Entropy. Procedia Computer Science, 151, 1046-1051. https://doi.org/10.1016/j.procs.2019.04.148

Min, T., Niu, L.-F., Feng, X.-y., Yi, Y., Wang, L.-m., Zhao, Y., \& Wang, H.-x. (2021). The effects of different temperatures on the storage characteristics of lotus (Nelumbo nucifera G.) root. Food Chemistry, 348, 129109. https://doi.org/10.1016/j.foodchem.2021.129109

Mirante, E., Georgiev, M., \& Gotchev, A. (2011, 16-18 May 2011). A fast image segmentation algorithm using color and depth map. Paper presented at the 2011 3DTV Conference: The True Vision - Capture, Transmission and Display of 3D Video (3DTV-CON). https://doi.org/10.1109/3DTV.2011.5877227

Momin, M. A., Rahman, M. T., Sultana, M. S., Igathinathane, C., Ziauddin, A. T. M., \& Grift, T. E. (2017). Geometry-based mass grading of mango fruits using image processing. Information Processing in Agriculture, 4(2), 150-160. https://doi.org/10.1016/j.inpa.2017.03.003

Park, J. J., \& Lee, W. Y. (2020). Softening of lotus root and carrot using freeze-thaw enzyme infusion for texture-modified foods. Food Bioscience, 35, 100557. https://doi.org/10.1016/j.fbio.2020.100557

Ramirez-Paredes, J.-P., \& Hernandez-Belmonte, U.-H. (2019). Visual quality assessment of malting barley using color, shape and texture descriptors. Computers and Electronics in Agriculture, 105110. https://doi.org/10.1016/j.compag.2019.105110

Rogowska, J. (2000). Overview and fundamentals of medical image segmentation. Handbook of medical imaging, processing and analysis, 69-85. https://doi.org/10.1016/B978-012077790-7/50009-6

Roy, K., Chaudhuri, S. S., Bhattacharjee, S., Manna, S., \& Chakraborty, T. (2019, 18-20 March 2019). Segmentation Techniques for Rotten Fruit detection. Paper presented at the 2019 International Conference on Opto-Electronics and Applied Optics (Optronix). https://doi.org/10.1109/OPTRONIX.2019.8862367

Ruedt, C., Gibis, M., \& Weiss, J. (2020). Quantification of surface iridescence in meat products by digital image analysis. Meat Science, 163, 108064. https://doi.org/10.1016/j.meatsci.2020.108064

Shafait, F., Keysers, D., \& Breuel, T. (2008). Efficient implementation of local adaptive thresholding techniques using integral images (Vol. 6815): SPIE. https://doi.org/10.1117/12.767755

Shao, D., Xu, C., Xiang, Y., Gui, P., Zhu, X., Zhang, C., \& Yu, Z. (2019). Ultrasound image segmentation with multilevel threshold based on differential search algorithm. IET Image Processing, 13(6), 998-1005. https://doi.org/10.1049/iet-ipr.2018.6150

Shrivakshan, G., \& Chandrasekar, C. (2012). A comparison of various edge detection techniques used in image processing. International Journal of Computer Science Issues 


\section{IMacrothink}

(IJCSI), 9(5), 269.

Sikora, T., \& Strada, A. (2005). Safety and quality assurance and management systems in food industry: An overview. The Food Industry in Europe, Agricultural University of Athens, Ateny.

Vafeiadis, T., Dimitriou, N., Ioannidis, D., Wotherspoon, T., Tinker, G., \& Tzovaras, D. (2018). A framework for inspection of dies attachment on PCB utilizing machine learning techniques. Journal of Management Analytics, 5(2), 81-94. https://doi.org/10.1080/23270012.2018.1434425

Vision, M. (2019). Improved Automatic Quality Inspections Through the Integration of State-of-the-Art Machine Vision and Collaborative Robots. Paper presented at the Advances in Manufacturing Technology XXXIII: Proceedings of the 17th International Conference on Manufacturing Research, incorporating the 34th National Conference on Manufacturing Research, 10-12 September 2019, Queen's University, Belfast.

Vitzrabin, E., \& Edan, Y. (2016). Adaptive thresholding with fusion using a RGBD sensor for red sweet-pepper detection. Biosystems Engineering, 146, 45-56. https://doi.org/10.1016/j.biosystemseng.2015.12.002

Wang, J., He, J., Han, Y., Ouyang, C., \& Li, D. (2013). An Adaptive Thresholding algorithm of field leaf image. Comput. Electron. Agric., 96, 23-39. https://doi.org/10.1016/j.compag.2013.04.014

Zemmour, E., Kurtser, P., \& Edan, Y. (2019). Automatic parameter tuning for adaptive thresholding in fruit detection. Sensors, 19(9), 2130. https://doi.org/10.3390/s19092130

\section{Copyright Disclaimer}

Copyright for this article is retained by the author(s), with first publication rights granted to the journal.

This is an open-access article distributed under the terms and conditions of the Creative Commons Attribution license (http://creativecommons.org/licenses/by/4.0/). 